Reprod. Nutr. Dévelop., 1982, 22 (6), 881-888.

\title{
Excrétion urinaire des glucides neutres et des glycoprotéines chez le veau préruminant recevant un aliment d'allaitement contenant de la farine de féverole
}

\author{
J. M. BESLE, M. DESRUT, P. THIVEND
}

Laboratoire de la Digestion des Ruminants, I.N.R.A.

Theix 63110 Beaumont, France

Summary. Urinary secretion of neutral carbohydrates and glycoproteins in the preruminant calf fed a milk replacer containing field bean.

In a previous work (Besle, Lassalas and Thivend, 1981), we showed the disappearance of 40 p. 100 of field bean $\alpha$-galactosides in the preruminant small intestine of the calf. To better understand the possible modification of absorption induced by antinutritional dietary factors with this type of feed, we measured the urinary excretion of neutral carbohydrates, glycoproteins and peptides in two preruminant calves receiving a milk replacer containing 31.5 p. $100 \mathrm{DM}$ of steam-flaked field bean. When the calves were 45 and 55 days old, the urine was collected quantitatively for 11 post-prandial hours or for 24 hrs.

Mono, di and tri-saccharides were determined by ion-exchange chromatography. Free and conjugated polysaccharides were separated by molecular sieve chromatography (MSC). The effluent was divided into three parts (fig. 1) which were used to assess total sugars, reducing sugars and proteins.

The main neutral carbohydrates excreted in urine (table 1) were (1) $\alpha$-galactosides and sucrose, (2) lactose, (3) starch breakdown products and (4) monosaccharides. The total amounts of $\alpha$-galactosides and sucrose excreted did not exceed $0.5 \mathrm{~g} /$ day $(2.1 \mathrm{p} .100$ intake), and their proportion (percentage of intake) varied inversely according to their molecular weight $(0.6$ to 3.5 p. 100 , respectively for ajugose and sucrose). Lactose was only slightly excreted $(0.2$ p. 100 of intake), starch breakdown products (maltotriose and tetraose) were excreted in small amounts $(0.01$ p. 100 of starch intake), and monosaccharides were mainly excreted as glucose.

For calves receiving field bean, the amounts of $\alpha$-galactosides (excluding raffinose) and sucrose excreted in urine (as a percentage of intake) were 2 to 3 times higher than in man or rat receiving these oligosaccharides in purified form. This could be due to the deleterious effects on absorption provoked by vicine and $\beta$-convicine in field bean. However, this hypothesis must be investigated further. Even taking into account the slight hydrolysis of these carbohydrates in the kidneys, absorption evaluated as urinary excretion was low compared to their intestinal disappearance ( 40 p. 100 of intake). Thus, we can assume that a large amount of $\alpha$-galactosides and sucrose is fermented by microorganisms in the small intestine.

Glycoproteins and peptides, separated by MSC into 14 fractions (fig. 1), amounted to a daily excretion of $14 \mathrm{~g}$ of protein and $0.8 \mathrm{~g}$ of conjugated carbohydrate (table 2). Some compounds contained a high carbohydrate fraction which was probably branched, while the others could have been pure peptides. A part of the chromatogram (peaks $L, W, Y$ ), quite similar to that obtained by Miettinen (1963) in human urine, is a rather complex mixture whose variations might indicate pathological conditions. 


\section{Introduction.}

Lors d'un travail précédent (Besle, Lassalas et Thivend, 1981) nous avons constaté que chez le veau préruminant recevant de la féverole, une fraction importante des $\alpha$-galactosides et du saccharose disparaissait dans l'intestin grêle. Cette disparition ne peut être causée ni par une hydrolyse acide dans la caillette (Täufel et al., 1964), ni par une glycolyse due aux enzymes de l'animal, celui-ci étant dépourvu d'enzymes spécifiques pour hydrolyser ces glucides. On ne peut donc l'expliquer que par une absorption directe et (ou) par une dégradation microbienne. II est bien connu que la féverole contient de nombreux composés toxiques dont certains sont détruits par la chaleur (facteurs antitrypsiques et antihémaglutiniques) alors que d'autres résistent à ce traitement, en particulier des globulines, comme la vicine et la $\beta$-convicine. Ces derniers composés sont responsables du favisme chez l'homme; ils provoquent chez le veau une réaction antigénique ainsi qu'une altération de la paroi intestinale, comme cela a été montré avec un régime contenant du soja (Kilshaw et Sissons, 1979 ; Barratt, Strachan et Porter, 1979). Nous avons pensé que ce phénomène pouvait être responsable d'une absorption importante des oligosides et expliquerait leur présence dans les urines. De plus, nous ignorons l'importance et la nature des autres composés glucidiques éliminés de cette manière.

Le but de cette expérience a donc été d'une part de mesurer l'excrétion urinaire des oligosides neutres non métabolisés et, d'autre part, d'apporter quelques éléments sur celle des glycoprotéines.

\section{Matériel et méthodes.}

Animaux, régimes, prélèvements. - Deux veaux Frisons mâles ont reçu un aliment d'allaitement contenant 31,5 p. 100 de féverole floconnée. Cet aliment, identique à celui décrit dans un de nos précédents travaux (Besle, Lassalas et Thivend, 1981) contenait 15 p. 100 d'amidon, 1,8 p. 100 de saccharose et 1 p. $100 \mathrm{~d}$ d' $\alpha$-galactosides. L'aliment d'allaitement a été reconstitué à raison de $130 \mathrm{~g} / \mathrm{kg}$ de lait distribué ; les quantités offertes ont augmenté avec le poids métabolique $\left(57 \mathrm{~g} / \mathrm{kg} \mathrm{P}^{0,75}\right)$ et ont été distribuées au seau 2 fois par jour, à $8 \mathrm{~h} 30$ et $17 \mathrm{~h} 00$. Après un nettoyage soigné de la région ventrale, les veaux ont été munis d'un dispositif de collecte de l'urine dont le fond était relié par un tuyau à deux flacons placés en série (dont un de garde) connectés à une pompe aspirante.

L'urine a été collectée en continu durant deux périodes de mesure aux âges de 45 et 55 jours. La première a été réalisée de 8 à $19 \mathrm{~h}$, les animaux ne recevant le second repas qu'après la fin du prélèvement; la seconde a duré $24 \mathrm{~h}$ à partir de $8 \mathrm{~h}$. Toutes les $3 \mathrm{~h}$ (sauf la nuit), le volume d'urine collecté était mesuré, les fermentations étaient stoppées par l'addition d'une solution de $\mathrm{HgCl}_{2} 5$ p. 100 $(\mathrm{P} / \mathrm{V})$ à raison de $20 \mathrm{ml} / \mathrm{l}$, puis le mélange conservé à $-20^{\circ} \mathrm{C}$.

Méthodes d'analyse. - Les protéines solubles ont été dosées suivant la méthode de Lowry et al. (1951), modifiée par Besle et al. (1983). Les glucides ont 
été dosés globalement (sucres totaux et réducteurs), ainsi que par chromatographie échangeuse d'ions (CEI) et tamis moléculaire (CTM), selon les méthodes décrites par Besle, Lassalas et Thivend (1981).

En sortie de colonne CTM, une partie de l'éluat était collecté sur fractionneuse, l'autre partie était divisée en 3 flux pour le dosage simultané des protéines solubles, des sucres totaux et des sucres réducteurs. Nous présentons ici les résultats obtenus sur les échantillons moyens d'urine réalisés pour chaque période et chaque animal.

\section{Résultats et discussion.}

L'urine contient quatre fractions glucidiques neutres ( $\alpha$-galactosides, saccharose, oligosides réducteurs, oses libres). On trouve en outre des glycoprotéines et quelques peptides (et/ou composés donnant une coloration avec le réactif de Lowry) qui ne semblent pas liés à des glucides. La totalité des osides liés et libres excrétés est d'environ $5 \mathrm{~g} / \mathrm{j}$, alors que celle de l'équivalent protéique est de $20 \mathrm{~g} / \mathrm{j}$.

Les quantités excrétées d' $\alpha$-galactosides (tabl. 1) atteignent en moyenne $165 \mathrm{mg} / \mathrm{j}$, soit 1,6 p. 100 des quantités ingérées (Ql). Cette proportion varie peu suivant I'animal, que ce soit sur 11 h (1 repas) ou sur 24 h (2 repas). L'absorption relative des $\alpha$-galactosides augmente lorsque le poids moléculaire diminue (de 0,6 pour l'ajugose à 3,5 p. 100 des QI pour le raffinose). Le saccharose est absorbé et excrété comme le raffinose, ce qui porte la quantité totale excrétée des glucides non réducteurs à environ $0,5 \mathrm{~g} / \mathrm{j}$.

L'absorption des $\alpha$-galactosides, distribués purs, a déjà été observée. Krause et al. (1968) constatent aussi, chez le rat comme chez l'homme, qu'elle est inversement proportionnelle au poids moléculaire. Mis à part le cas du raffinose qui a pu être excrété au taux de 5 p. 100 des Ql chez le rat (Krause et al., 1968), nos résultats d'excrétion, relatifs aux quantités ingérées, chez le veau, sont en moyenne 2 à 3 fois supérieurs à ceux obtenus chez le rat et sont nettement plus élevés que ceux mesurés chez l'homme lexcrétion de moins de 1 p. 100 des $\alpha$ galactosides ingérés selon Täufel et al., 1965). II y aurait sans doute un effet animal, le veau jeune absorberait plus de grosses molécules que l'homme ou le rat, mais on peut suggérer une influence de certains composés (vicine et $\beta$-convicine) de la féverole qui pourraient modifier l'absorption. Une $\alpha$-galactosidase rénale a été mise en évidence chez le ruminant par Debris, Courtois et Petek (1962). Elle hydrolyse légèrement le raffinose et très peu le stachyose pour libérer du galactose. De ce fait, il est possible que les quantités $d^{\prime} \alpha$-galactosides et de saccharose absorbées soient légèrement supérieures à celles mesurées par l'excrétion urinaire (2,1 p. 100 des Ql). Même dans ce cas, elles ne représentent qu'une petite partie de celles qui ont disparu dans l'intestin grêle (40 p. 100 des Ql). On peut donc penser que la majeure partie de la dégradation de ces oligosides est due à l'action des microorganismes. On sait en effet que l'intestin grêle du veau contient une microflore abondante (Contrepois et Gouet, 1973) dont l'activité mesurée par la nature et la concentration des produits terminaux de la fermenta- 


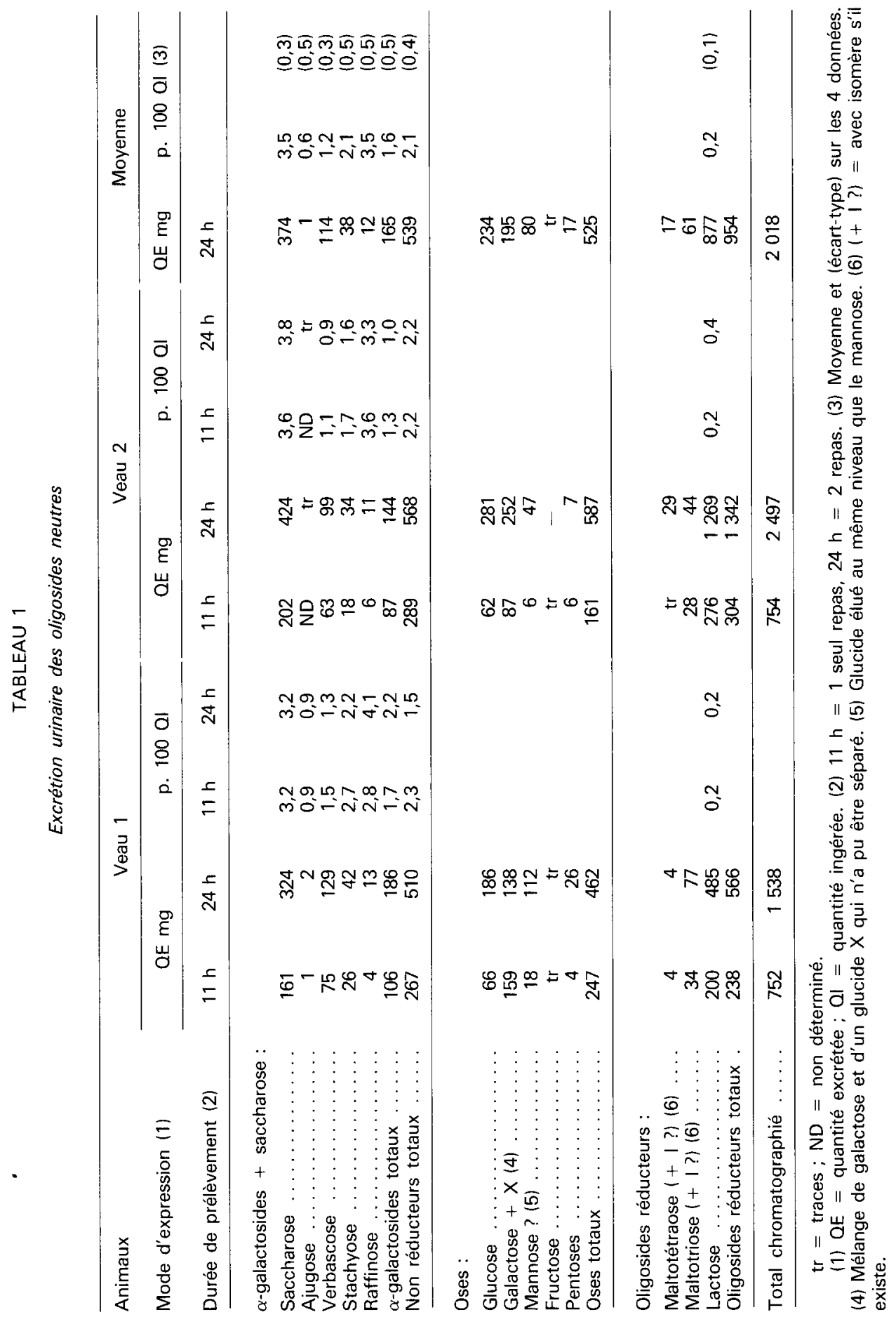


tion microbienne (acides gras volatils, acide lactique, azote ammoniacal) est importante chez les veaux recevant de la féverole (Besle, Lassalas et Thivend, 1981).

Nous avons vérifié par $\mathrm{CEl}$ que le seul diholoside réducteur excrété est le lactose en quantité moyenne de $877 \mathrm{mg} / \mathrm{j}$, soit seulement $0,2 \mathrm{p} .100$ de la quantité ingérée. Ce résultat est proche de celui trouvé par Rutloff et al. (1967) chez I'homme (0,33 p. 100 des Ql). Nous n'avons pas détecté de maltose, d'une part, parce qu'il est hydrolysé dans l'intestin et, d'autre part, parce qu'il peut être métabolisé dans l'organisme après absorption (Weser et Sleisenger, 1967).

Les oligosides réducteurs isolés sont constitués d'une faible quantité de maltotriose et de maltotétraose (en moyenne respectivement 60 et $16 \mathrm{mg} / \mathrm{j}$ ) correspondant à 0,01 p. 100 de I'amidon ingéré. Au total l'augmentation de l'excrétion en sucres réducteurs provoquée par la présence de l'amidon dans le régime, déjà notée par Mathieu, Thivend et Barre (1970) n'est que très légère dans le cas de la féverole (en moyenne 1,7 p. 100 du pouvoir réducteur total).

Le principal ose dosé, le glucose, est présent en quantité normale $(233 \mathrm{mg} / \mathrm{l})$. Le galactose semble être excrété en quantité anormalement abondante, mais il n'a pu être suffisamment différencié d'un glucide $X$ pour être quantifié avec certitude. II pourrait provenir de la coupure des $\alpha$-galactosides par l' $\alpha$-galactosidase rénale. Les pentoses, présents en petite quantité, ont été différenciés en 2 composés, élués en même temps que le ribose et le xylose.

TABLEAU 2

Excrétion urinaire des peptides et des glycoprotéines

(Quantités excrétées en mg par jour par 2 veaux âgés de 55 j)

Dosage global des glucides et des protéines

\begin{tabular}{|c|c|c|c|c|c|}
\hline \multicolumn{2}{|c|}{ Composé } & \multicolumn{2}{|c|}{ Veau 1} & \multicolumn{2}{|c|}{ Veau 2} \\
\hline Appellation (1) & Nature & glucide (2) & peptide $\{3\}$ & glucide (2) & peptide 13 \\
\hline Y (exclusion) & glycoprotéine(s) & 204 & 1771 & 245 & 1696 \\
\hline$v+w$ & पitude & 29 & 798 & 24 & 355 \\
\hline$L B(4)+T$ & - & 345 & 1045 & 603 & 1051 \\
\hline $\mathrm{L}$ & - & 53 & 7439 & 86 & 7660 \\
\hline$J+k$ & - & 9 & ND & 11 & 86 \\
\hline $\mathrm{H}$ & - & 9 & 59 & 8 & 75 \\
\hline G & _- & -_- & - & 7 & 69 \\
\hline $\mathrm{D}$ & peptides? & $\operatorname{tr}$ & 81 & ND & 157 \\
\hline $\mathrm{c}$ & - & $\operatorname{tr}$ & 1008 & ND & 546 \\
\hline $\mathrm{B}_{1}+\mathrm{B}_{2}+\mathrm{B}_{3}$ & - & tr & 966 & ND & 1327 \\
\hline $\begin{array}{lll}1 & A_{3}^{2}\end{array}$ & glycopeptide & 11 & 620 & 11 & 935 \\
\hline & Total & 660 & 13687 & 995 & 13957 \\
\hline
\end{tabular}

Dosage global

Equivalent glucidique $\times 0,9$ ou protéique (5)

4971

18160

6267

20777

(1) Pics correspondants à un volume d'élution déterminé. Voir figure 1. (2) Evalués en équivalents « dextrines ». (3) Evalués en équivalents albumine de sérum bovin. (4) Correction de dérive de ligne de base car les pics sont mal séparés (voir fig. 1). (5) Dosage des sucres totaux par rapport au glucose, corrigé $(\times 0,9)$ pour tenir compte de la polymérisation; dosage des protéines totales par rapport à l'albumine de sérum bovin. 
Nous avons pu discerner 14 pics correspondant à des composés protéiques (fig. 1), certains étant très mal séparés ; 10 des principaux d'entre eux ont pu cependant être quantifiés. Certains contiennent une proportion importante de glucides, en général réducteurs (niveaux $A 3, G, H, K, L, W, Y$ ), d'autres donnent une coloration avec le réactif de Lowry (peptides et autres composés) sans contenir de fraction glucidique détectable (niveaux B, C, D). Dans ce travail, nous admettons que la coïncidence des pics protéines-glucides correspond à une glycoprotéine ou à un mélange oligoside(s)-glycoprotéine(s) ; la fraction glucidique sera appelée "glucides liés». La totalité de ceux-ci atteint en moyenne $0,8 \mathrm{~g} / \mathrm{j}$ pour $14,6 \mathrm{~g} / \mathrm{j}$ d'équivalent protéique.

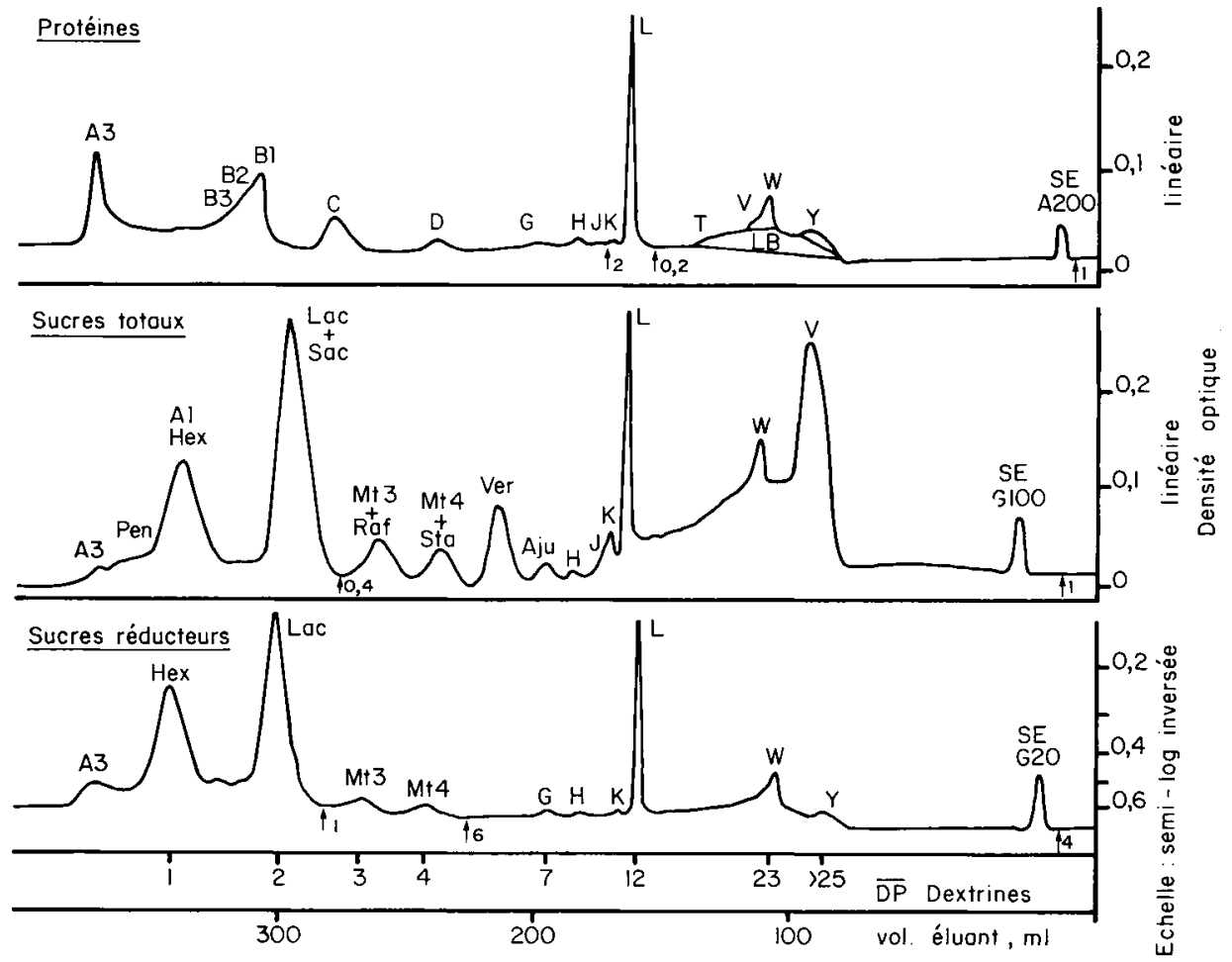

FIG. 1. - Exemple de séparation par tamisage moléculaire sur Biogel (CTM) des glucides et des g/ycoprotéines urinaires. Veau 2, 55 jours, échantillon moyen. $A_{3} \ldots Y=$ niveaux du chromatogramme correspondant aux DP des dextrines.

Pen $=$ Pentoses (niveau $A_{2}$ ), Hex $=$ Hexoses (niveau $A_{1}$ ), Lact $=$ Lactose, Sac $=$ Saccharose, $\mathrm{Mt3}=$ maltotriose, Raf $=$ Raffinose, $\mathrm{Mt4}=$ Maltotétraose, Sta $=$ Stachyose, Verb $=$ Verbascose, Aju = Ajugose, SE - A200 = Standard externe albumine $200 \mathrm{~g} / \mathrm{ml}, \mathrm{SE}-\mathrm{G} 100=$ Standard externe glucose $100 \mathrm{~g} / \mathrm{ml}$, SE-G20 = Standard externe glucose $20 \mathrm{~g} / \mathrm{ml}$ DP dextrines = Degré de polymérisation calculé pour un mélange de chaînes de dextrines non ramifiées. 
Le pouvoir réducteur des fractions $L, W, Y$ est bien supérieur à celui que l'on obtiendrait avec une chaîne glucidique liée linéaire, la différence pouvant provenir en partie du groupement protéique (fonctions thiols) mais aussi principalement de chaînes osidiques ramifiées. Chez l'homme, une telle structure a été isolée par Strecker et Lemaire-Poiteau (1977); en outre, la séquence L, W, Y ressemble beaucoup à celle décrite par Miettinen (1963). Le même nombre de composés protéiques a été séparé par Pechan (1964) sur charboncélite, mélange qui a été décomposé encore en 45 oligosides et oligopeptides par Strecker et Lemaire-Poiteau (1977). Enfin, des peptides libres (tels que sans doute ceux des niveaux B, C, D) ont été mis en évidence par Meilman, Urivetzky et Rapoport (1963).

En conc/usion, nous avons montré que le veau est capable d'absorber dans l'intestin grêle et d'excréter dans l'urine des molécules glucidiques aussi longues que l'ajugose. Lorsque cet animal reçoit de la féverole, l'excrétion des $\alpha$ galactosides paraît supérieure à celle observée chez d'autres espèces recevant ces glucides purifiés. II est possible que les substances antinutritionnelles de l'aliment modifient l'absorption; toutefois il serait intéressant de faire la comparaison dans des conditions expérimentales semblables. L'absorption des $\alpha$ galactosides et du saccharose n'est tout de même pas suffisante pour expliquer la disparition de 40 p. 100 d'entre eux dans l'intestin grêle. Ces composés seraient donc principalement décomposés par la microflore.

Le diagramme des glycoprotéines séparées par CTM, est proche de celui observé chez d'autres espèces : il correspond à un mélange de nombreux composés qu'il serait intéressant de purifier et d'étudier pour en connaître la structure et l'origine physiologique. Ce travail, mené chez l'homme par Strecker et Lemaire-Poiteau (1977) est toutefois très complexe mais intéressant puisque la connaissance globale du diagramme des glycoprotéines peut suffire pour mettre en évidence des états pathologiques (Miettinen, 1963 ; Strecker et Montreuil, 1979).

Reçu en février 1982 Accepté en mai 1982

\section{Références}

BARRAT M. E. J., STRACHAN P. J., PORTER P., 1979. Immunologically mediated nutritional disturbances associated with soya-protein antigens. Proc. Nutr. Soc., 38, 143-150.

BESLE J. M., LASSALAS B., THIVEND P., 1981. Digestion des glucides de la féverole par le veau préruminant. Reprod. Nutr. Dévelop., 21, 629-649.

BESLE J. M., POUGHEON M., BAYLE E., THIVEND P., 1983. Etude des glucides intestinaux et des glycoprotéines d'origine endogène chez le veau préruminant. Reprod. Nutr. Dévelop. 23. (sous presse).

CONTREPOIS M., GOUET P., 1973. La microflore du tube digestif du jeune veau préruminant ; dénombrement de quelques groupes bactériens à différents niveaux du tube digestif. Ann. Rech. vét., 4, 161-170. 
DEBRIS M. M., COURTOIS J. E., PETEK F., 1962. Sur quelques caractères de l' $\alpha$-galactosidase rénale. Bull. Soc. Chim. biol., 44, 291-299.

KILSHAW P. J., SISSONS J. W., 1979. Gastrointestinal allergy to soybean protein in preruminant calves. Antibody production and digestive disturbances in calves fed heated soya bean flour. Res. vet. Sci., 27, 361-365.

KRAUSE W., TÄUFEL K., RUTTLOF H., MAUNE R., 1968. Zur enzimatischen Spaltung und Resorption von $\mathrm{Di}$ - und höheren Oligo sacchariden im Intestinaltrakt von Tier und Mensh. Ernährungsforschung, 13, 161-169.

LOWRY O., ROSEBROUGH N. J., FARR A. L., RANDALL X. J., 1951. Protein measurement with the Folin phenol reagent. J. biol. Chem., 193, 265-275.

MATHIEU C. M., THIVEND P., BARRE P. E., 1970. Digestion et utilisation des aliments par le veau préruminant à l'engrais. $\mathrm{V}$ - Remplacement des matières grasses du lait par des dextrines. Ann. Biol. anim. Bioch. Biophys., 10, 253-269.

MEILMAN E., URIVETZKY M. M., RAPOPORT C. M., 1963. Urinary hydroxyproline peptides. J. clin. Invest., 42, 40-50.

MIETTINEN T. A., 1963. Further studies on gel filtration of urinary mucosaccharides. Clin. chim. Acta., 8, 693-700.

PECHAN Z., 1964. Hexosamine containing substances and cancer. II. Isolation and characterization of hexosamine containing low molecular weight from human urine. Neoplasma, 11, 279-291.

RUTTLOFF H., TÄUFEL A., KRAUSE W., HAENEL H., TÄUFEL K., 1967. Die intestinalenzymatische Spaltung von Galacto-Oligosacchariden im Darm von Tier und Mensch mit besonderer Berücksichtigung von Lactobacillus bifidus. 2 Mitt. Zum intestinalen Verhalten der Lactulose. Nahrung, 11, 39-46.

STRECKER G., LEMAIRE-POITEAU A., 1977. Fractionation and characterization of acidic oligosaccharides and glycopeptides from normal and pathological urines. J. Chromatog., 143, 553-569.

STRECKER G., MONTREUIL J., 1979. Glycoprotéines et glycoprotéinoses. Biochimie, 61, 1199-1246.

TÄUFEL A., KRAUSE W., RUTTLOFF H., TÄUFEL K., 1964. Intestinale Resorption und Ausscheidung von Raffinose. Nahrung, 8, 107-108.

TÄUFEL K., RUTTLOFF H., KRAUSE W., TÄUFEL A., VETTER K., 1965. Zum intestinalen Verhalten von Galakto-Oligosacchariden beim Menschen. Klin. Wschr., 43, 268-272.

WESER E., SLEISENGER M. H., 1967. Metabolism of circulating dissacharides in man and the rat. J. clin. Invest., 46, 499-505. 